\title{
New method of apertured electromagnetic field modeling
}

\author{
S. Anokhov', A. Khizhnyak ${ }^{2}$, R. Lymarenko ${ }^{1}$ \\ ${ }^{\prime}$ International Center "Institute of Applied Optics", National Academy of Science of Ukraine, 10-G Kudryavska street, 04053 Kyiv, \\ Ukraine, phone: 380(44)2122158, fax 380(44)2124812,e-mail: iao@i.kiev.ua \\ ${ }^{2}$ Tellabs Operations, Inc., 15 Skyline Dr., Hawthorne, NY, 10532, USA
}

\begin{abstract}
Using a new wave treatment of rigorous Sommerfield's solution for a problem of plane wave diffraction on a perfectly conductive half-plane, it was obtained the solution for a problem of plane wave diffraction on a slit and rectangular aperture. The result of aperture diffraction was represented as a sum of elementary rectangular unit cell waves. New integral approach to modeling of plane wave diffraction on an arbitrary two-dimensional aperture is discussed. Proposed method is very useful for providing numerical modeling of diffraction phenomena.
\end{abstract}

Keywords: diffraction, two-dimensional aperture, computer modeling.

Paper received 11.09.01; revised manuscript received 24.10.01; accepted for publication 12.12.01.

\section{Introduction}

The development of optics, optical engineering and optical measurements determines the necessity to provide more exact modeling of diffraction effects, because of the traditional methods of their description appear to be unsatisfactory. In this connection, the results of further development of the approach that based on new wave interpretation of a rigorous Sommerfield's solution are represented. A new treatment of the well-known Sommerfeld's solution [1,2] of the problem of plane-wave diffraction from a perfectly conducting half-plane is good proofground for further more deep investigation of diffraction field and can be generalized on cases of apertured electromagnetic wave diffraction.

As it was shown in our previous papers [3], in both theory and experiment, the diffraction field can represented as a superposition of real physically existing waves can propagate in free space separately with self-similar features, in contrast to geometrical and boundary waves postulated in traditional representation. The introduced singular wave component - edge dislocation wave (EDW), was described by the complex Fresnel integral and possess the main numerical information about diffraction process.
The aim of this work is an attempt to generalize the new approach to more complex apertures, in particular, to the slit and rectangular ones. Also, it was consider the development of the original method of field modeling, which was formed using plane wave diffraction on a two-dimensional aperture.

\section{EDW as «eigenmode» for diffraction field representation}

\section{a. New decomposition of plane wave diffraction on two orthogonal half-planes.}

Peculiarity of the EDW is a scale-invariant space behavior regarding the $U$ parameter and the edge dislocation that presents on the boundary of geometrical shadow. This is a reason for naming this wave as Edge Dislocation Wave. Symmetrical structure and the analytical form in whole space allow to treat EDW as a basic function for diffraction field representation. It is well-known that the arbitrary wave was represented as an angular spectrum of plane waves. This allows to use the solution of model problem of plane wave 


\section{S. Anokhov et al.: New method of apertured electromagnetic...}

diffraction for constructing the solution of the problem of arbitrary beam diffraction. The beam, in particular, the Hermit-Gaussian one, diffraction on a half-plane was considered in [4], where it was shown that, in the problem of diffraction, the edge dislocation wave plays the role of an «eigenmode» like that of the plane wave in free space. Due to this, the solving of the mentioned problem is reduced to calculation of convolution of a source beam angle spectrum and introduced edge dislocation wave that composed the singular component of diffraction beam.

Next step toward description of diffraction on two-dimensional aperture consists of consideration of plane wave diffraction on a rectangular aperture. From the very beginning, we consider a rectangular sector that is composed of two orthogonal half-planes. Let a plane wave $E_{0}$ propagates along Z-axes and is incident normally on a sector plane, the sides of which were oriented along $\mathrm{X}$ - and $\mathrm{Y}$-axes (Fig. 1). This process can be imagined that as a result of two sequential acts of diffraction on each of half-planes. In this case, the second half-plane was illuminated by $E_{d}$ wave that was denoted as $E_{0} \times\left(\xi \cdot f(U)+\frac{1}{2}\right)$, where the constant $\xi=\frac{1-i}{\sqrt{2 \pi}}$, and the function $f(U)=\int \exp \left(i \mu^{2}\right) d \mu$ is the well-known Fresnel integral in the complex form. The parameter $U$ for normal incidence can be written as $U= \pm \sqrt{k} \sqrt{\sqrt{x^{2}+z^{2}}-z}$, the sign «-» is used for $x<0, z$ $>0, \mathrm{k}$ is a wave-vector. Because of the angular spectrum $F(k)$ of the wave, that is not incident on the second halfplane, was represented as delta-function on $y$-coordinate, the resulting diffraction field is reduced to the product of a transmission function of two orthogonal half-planes:

$E^{o r t}=E_{0} \times\left(\xi \cdot f\left(U_{x}\right)+\frac{1}{2}\right) \cdot\left(\xi \cdot f\left(U_{y}\right)+\frac{1}{2}\right)$,

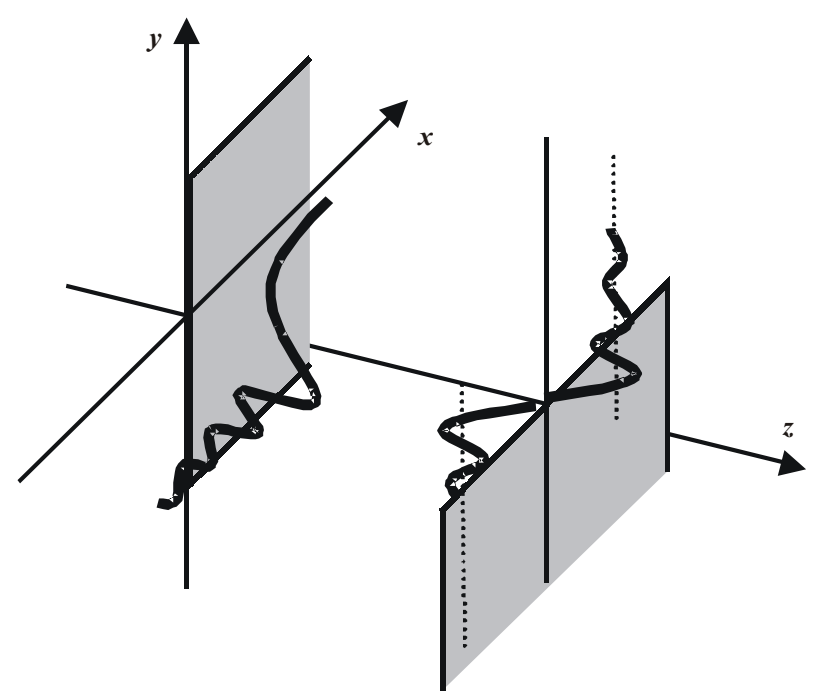

Fig. 1. Scheme of diffraction on orthogonal half-planes. where $U_{y}= \pm \sqrt{k} \sqrt{\sqrt{y^{2}+z^{2}}-z}$. In Eq. (1), the mirror reflected component was omitted, which corresponds to a solution for a "black" screen that is an essentially scalar problem.

\section{b. Plane wave diffraction on a rectangular aperture}

The field of plane wave diffraction on a slit in perfectly conductive plane can be written in the form [8]:

$E^{s l i t}=E_{0} \frac{1-i}{\sqrt{2 \pi}} \int_{U_{1}}^{U_{2}} \exp \left(i \mu^{2}\right) d \mu$

where $a$ is a half-width of a slit, $U_{1}= \pm \sqrt{k} \sqrt{\sqrt{(x-a)^{2}+z^{2}}-z}$, $U_{2}= \pm \sqrt{k} \sqrt{\sqrt{(x+a)^{2}+z^{2}}-z}$, sign " + " is used for $x-a>$ $0(x+a>0)$ and otherwise. This result can be easily generalized on arbitrary angle of plane wave incidence [1,8]. Exp. (2) explains the two EDWs that were born on each side of a slit. Using the Exp. (2) we obtain the resulting field for plane wave diffraction on a rectangular aperture $2 a \times 2 b$ centered on the origin of coordinates (Fig. 2). The diffraction field $E$ we explain using transmittance $\mathrm{W}$ that was product on initial plane wave $E_{0}: E=W \times E_{0}$.

Using the expression (2) for slit diffraction field we obtain the expression for plane wave diffraction on a rectangular aperture. Thus, the coefficient $W^{l}$ can be expressed via orthogonal EDW-functions $f_{x}$ and $f_{y}$, which are born on each side of the rectangular:

$W=\frac{i}{\pi}\left\{f\left(U_{x}(x-a)\right)-f\left(U_{x}(x+a)\right)\right\} \times$

$\times\left\{f\left(U_{y}(y-b)\right)-f\left(U_{y}(y+b)\right)\right\}$.

In a scalar non-paraxial diffraction theory, i.e. without «evanescent» wave [2], the result of diffraction of a plane wave on a rectangular aperture was written as a product of transmittance of two cross-orthogonal slits.

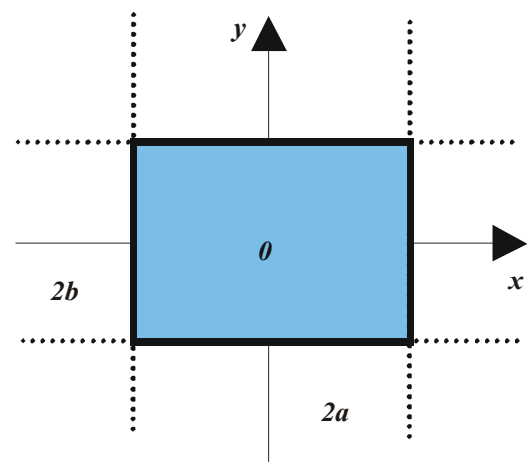

Fig. 2. Scheme of a diffraction on a rectangular aperture.

SQO, 4(4), 2001 


\section{S. Anokhov et al.: New method of apertured electromagnetic...}

\section{c. Diffraction on a linear element}

Now we consider the diffraction on a half-slit (Fig. 3), which can be treated as a limit case of the rectangular aperture:

$$
\begin{aligned}
& E=E_{0} \times \frac{i}{\pi}\left[f(U(x))+\frac{1}{2}\right] \times \\
& \times\left[f\left(U\left(y-\frac{\delta l}{2}\right)\right)-f\left(U\left(y+\frac{\delta l}{2}\right)\right)\right] .
\end{aligned}
$$

This allows us to find the field that was produced by the boundary linear element $d l$. Taking the limit in (4) and subtracting the part of the field that correspond to the slit (dotted line at $x>0$ on Fig. 3) we obtain that:

$$
W d l=\frac{i}{\pi} \exp \left(i U_{y}^{2}\right) \cdot \frac{\partial U_{y}}{\partial y} \cdot\left(f(U(x))-\frac{1}{2}\right) d l
$$

The experimental distribution of intensity of the field diffracted on half-and-squared cell was shown in Fig. 4. Single mode linearly polarized $\mathrm{He}-\mathrm{Ne}$ laser radiation was used to produce the nearly plane wave beam, and the diffraction field was registered using CCD camera. We see the approach to spherical symmetrical form of distribution during with decreasing the cell size. The structure

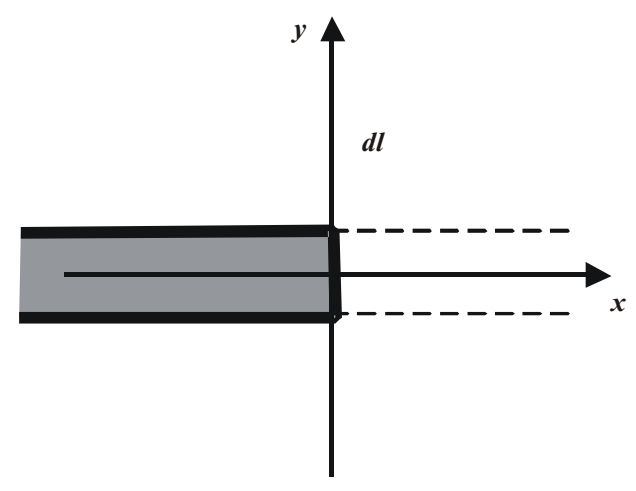

Fig. 3. Schematically view of the creation of boundary element $d l$.

peculiarity was visualized using subtraction of the plane wave with amplitude that was twice less than the incident wave.

\section{New scalar solution for problem of a plane wave diffraction on a two-dimensional aperture}

\section{a. Plane wave diffraction on a composed aperture}

Further we consider the plane wave diffraction on the aperture that composed of rectangular cells. In simple case of two rectangular cells A and B (Fig. 5) with one contiguous side, the diffraction field can be expressed as:
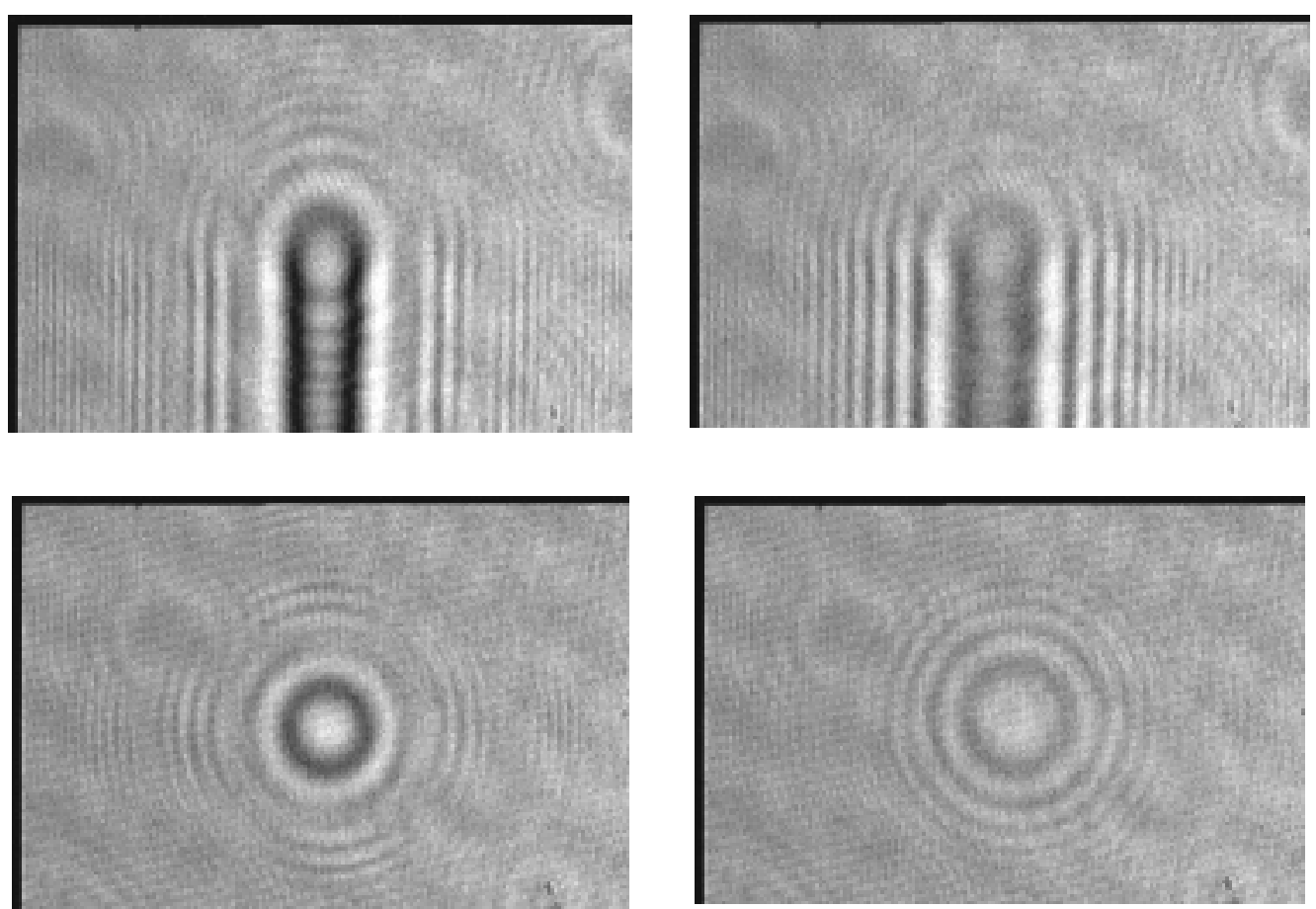

Fig. 4. The experimental frames of intensity distribution demonstrate the structure of elementary diffraction wave for half-slit and squared cell under the decreasing of its size. The structure detail is visualized by subtracting the incident plane wave with half-amplitude. Frame size is $4.8 \times 3.2 \mathrm{~mm}$. 


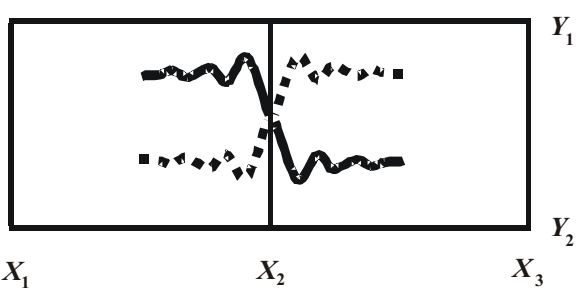

Fig. 5. The compensation of EDW oscillations from contiguous sides of rectangular cells.

$\sum_{n} W_{n}=\left(X_{1}-X_{2}\right)\left(Y_{1}-Y_{2}\right)+$

$+\left(X_{2}-X_{3}\right)\left(Y_{1}-Y_{2}\right)=\left(X_{1}-X_{3}\right)\left(Y_{1}-Y_{2}\right)$,

where $X_{i}$ and $Y_{i}$ are EDW-functions, correspond the sides that were shown in Fig. 5. I.e. the inner EDW (in this case $X_{2}$ ) does not take a part in the field construction. This peculiarity remains for more complex apertures that were composed of rectangular cells.

In contradiction to another method of the wave equation solution, the proposed integral representation of diffracted field was based on obtained solution of the problem of plane wave diffraction on rectangular aperture and slit. Thus, using the unit rectangular cell representation of twodimensional aperture, we obtain the diffraction field in the form:

$$
E=E_{0} \times \sum_{n} W_{n}
$$

It is easy to see that the EDW-function of inner unit cell doesn't take part in field construction. This peculiarity of introduced rectangular unit cell - inter-destruction of field oscillation from jointed inside is sufficient decrease the needed number of elementary cell in (7). This allows sufficiently decrease the time of computer modeling without losses of accuracy. The Fig. 6 shows the example of adaptive unit cell representation of aperture.

\section{b. Arbitrary aperture}

No doubt that the Exp. (7) is useful for apertures with stepped boundary, and so it is need to reduce the step for more precisely approximation of curvilinear apertures. Using the local coordinates, we can to easily obtain the expression for the case of the arbitrary angle of a plane wave incidence on two-dimensional apertures. All of W-waves is cophasal and differ from each other by only initial points.

Let's consider one of multipliers in Eq. (3) in more detail, using the Fresnel integral:

$$
\begin{aligned}
& \Delta W_{x}=\frac{1-i}{\sqrt{2 \pi}}\left\{\int_{0}^{U_{1}} \exp \left(i \mu^{2}\right) d \mu-\int_{0}^{U_{2}} \exp \left(i \mu^{2}\right) d \mu\right\}= \\
& =\frac{1-i}{\sqrt{2 \pi}} \int_{U_{2}}^{U_{1}} \exp \left(i \mu^{2}\right) d \mu
\end{aligned}
$$

where $U_{1}$ and $U_{2}$ are the coordinates of the observation point $\mathrm{P}$. When the size of cell is decreased, we suppose that $U_{2}=U_{1}+\Delta U$ and the difference of two Fresnel integrals can be written in the form:

$$
\begin{aligned}
& \Delta W_{x}=\frac{1-i}{\sqrt{2 \pi}}\left\{\int_{0}^{U+\Delta U} \exp \left(i \mu^{2}\right) d \mu-\int_{0}^{U} \exp \left(i \mu^{2}\right) d \mu\right\}= \\
& =\frac{1-i}{\sqrt{2 \pi}} \int_{U}^{U+\Delta U} \exp \left(i \mu^{2}\right) d \mu
\end{aligned}
$$

Although the $\Delta U$ depends on coordinates of the observation point and width of cell, it is necessary to use only the fact that $\Delta U$ trends to zero when the slit width does the same. This allows writing a limit for Exp. (9) in the form:

$$
\lim _{\Delta U \rightarrow 0} \Delta W_{x}=\frac{1-i}{\sqrt{2 \pi}} \cdot 2 \cdot \Delta U \cdot \exp \left(i U^{2}\right)
$$

In the limit case, when the size of unit cell trends to zero, we obtain the expression for secondary wave W (Unit Cell Wave), which is illuminated by elementary cell $d s=d \xi d \eta$, where $\xi$ and $\eta$ are coordinates on a screen plane (Fig. 7):

$$
E=E_{0} \times W=E_{0} \times \frac{i}{\pi} \cdot \Delta U_{x} \cdot \Delta U_{y} \cdot \exp \left\{i U_{x}^{2}+i U_{y}^{2}\right\}
$$

Taking into account that $\Delta U=\frac{\partial U}{\partial x} d x$, the expression

(7) can be written in the following integral form:

$$
\begin{aligned}
& E(x, y, z)=E_{0} \times \iint_{A} W d s= \\
& =E_{0} \times \iint_{A} \frac{i}{\pi} \frac{\partial U_{\xi}}{\partial \xi} \frac{\partial U_{\eta}}{\partial \eta} \exp \left(i U_{\xi}^{2}+i U_{\eta}^{2}\right) \times \\
& \times \exp (-i k z) d \xi d \eta
\end{aligned}
$$

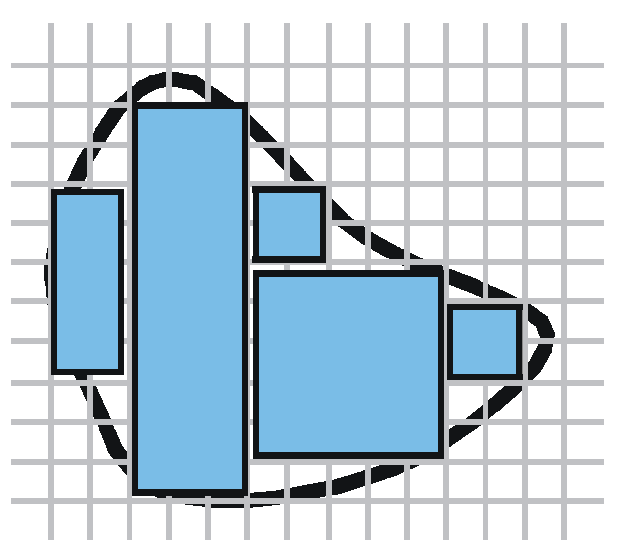

Fig. 6. Adaptive rectangular unit cell representation of two-dimensional aperture. 


\section{S. Anokhov et al.: New method of apertured electromagnetic...}

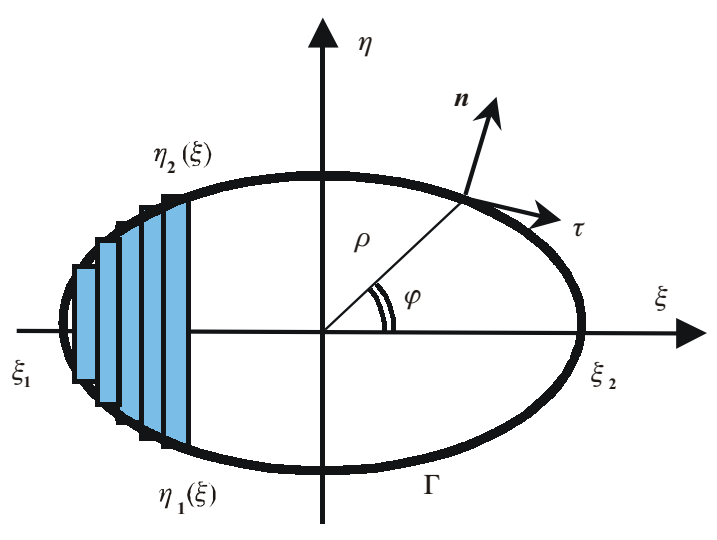

Fig. 7. Coordinate system in screen plane for simplifying the exp. (12).

where $U_{\zeta}$ and $U_{\eta}$ are dimensionless curvilinear quasi-parabolic coordinates: $U_{\xi}= \pm \sqrt{k} \sqrt{\sqrt{(x-\xi)^{2}+z^{2}}-z}$, $U_{\eta}= \pm \sqrt{k} \sqrt{\sqrt{(y-\eta)^{2}+z^{2}}-z}$ and the sign " + " is used for $x-\xi>0(y-\eta>0)$ and vice versa. In paraxial approximations, the Exp. (12) is transformed into the well-known Rayleigh-Sommerfeld solution for two-dimensional problems with Dirichlet conditions and Kirchhoff's approximation and also clearly illustrate the Huygens-Fresnel principle $[1,5,9,10]$.

The expression (12) gives us the non-paraxial scalar solution of the problem of plane wave diffraction on a perfectly conductive arbitrary two-dimensional screen. This expression is useful for analytical and numerical calculations. It should also be stated that the integral representation (12) describes not only forward-propagating component but also mirror-reflecting component of diffracted field with accounting the full field $[2,3]$ representation of the model problem.

It is surprising that the partial derivation $\frac{\partial U}{\partial x}=\sqrt{\frac{k}{2 r}} \cos \left(\frac{\theta}{2}\right)$ in Exp. (8) coincides with the elementary solution of the two-dimensional Helmholtz equation $[1,5]$. It is wonder that the Exp. (12) is still right in free space without screen. That is an argument to propound a question that an arbitrary wave can expand into W-representation for describing the light beam propagation in free space.

\section{c. Simplifying the field expression}

Due to a specific form of the convolution kernel, the aperture integral (12) can be reduced to multiple one and, moreover, to a simple expression using the symmetry of the considered problem. This specificity allows obtaining the solution in an analytical form for some cases. Let the aperture is given by continuous functions $\eta_{1}(\xi)$ and $\eta_{2}(\xi)$ (Fig. 7), then:

$$
\begin{aligned}
& E=E_{0} \times \int_{\xi_{1}}^{\xi_{2}} d \xi \int_{\eta_{1}(\xi)}^{\eta_{2}(\xi)} d \eta \cdot W= \\
& =E_{0} \times \int_{\xi_{1}}^{\xi_{2}} d \xi \frac{\partial U_{\xi}}{\partial \xi} \exp \left(i U_{\xi}^{2}\right)^{\eta_{2}} \int_{\eta_{1}(\xi)}(\xi) \frac{\partial U_{\eta}}{\partial \eta} \exp \left(i U_{\eta}^{2}\right)
\end{aligned}
$$

Only outside $\eta$-EDW is included into field expressions for vertical strip. This allows further simplification and the Exp. (6) can be rewritten using the adaptive like «wavelet» kernel:

$$
\begin{aligned}
& E=\frac{i}{\pi} \int_{\xi_{1}}^{\xi_{2}} d \xi \cdot \exp \left(i U_{\xi}^{2}\right) \frac{\partial U_{\xi}}{\partial \xi} \times \\
& \times\left[E D W_{\eta}\left(\eta-\eta_{2}(\xi)\right)-E D W_{\eta}\left(\eta-\eta_{1}(\xi)\right)\right]
\end{aligned}
$$

In some cases the Exp. (14) is more useful for analytical and numerical calculations then (12). This approach was used for modeling of the diffraction phenomena and studying the creation of optical vortices under diffraction on an elliptical aperture as well as the transformation of hidden dislocation trajectory under smooth deformation of aperture from the circular to elliptical one [7]. If an arbitrary aperture is defined by contour $\Gamma$, the diffraction field can be expressed as a contour integral by using the boundary element (5):

$$
E=\oint_{\Gamma} \frac{1}{2 \pi} \exp \left(i U_{\tau}^{2}\right) \cdot \frac{\partial U_{\tau}}{\partial \tau} \cdot \exp \left(i U_{n}^{2}\right) \frac{1}{2 U_{n}} d l+G
$$

where the diffraction field was represented traditionally [6] as sum of geometrical $G$ and boundary waves, $(n, \tau)$ is a normal and tangent to curve element $d l$ of aperture boundary $\Gamma$ (Fig. 7). Based on (15) it is more useful to consider the plane wave diffraction on the elliptical aperture in detail using the polar coordinates $(\rho, \varphi)$.

\section{Summary}

The new method of solution of the problem of plane wave diffraction on two-dimensional perfectly conductive aperture is considered. It is shown that the diffracted field can be represented via orthogonal EDW-functions, that play the role of eigenmodes like those of plane waves in free space. Developed on the base of proposed integral representation the effective algorithm is very useful for computer modeling the aperture diffracted field and allows eliciting features of spatial behavior. The totality of experimental results substantiated the theoretical conclusions. It was shown the possibility of sufficient decreasing the amount of numerical calculations without loss of generalities.

The solution for the problem of plane wave diffraction on orthogonal half-planes was obtained. This result was 
generalized on plane wave diffraction on an arbitrary two-dimensional aperture. It was shown that the total diffraction field is a sum of elementary waves from rectangular cells that composed the aperture. The fields that correspond to contiguous sides of the rectangular cell have a phase shift equal to $\pi$. The peculiarity of this approach results in a destructive interference between amplitude oscillations of fields in inner cells of aperture. It was shown that only outside cells take part in forming the diffraction field, and this fact can be treated as a way for jumping from aperture integral to the contour one. The specific form of the convolution kernel that describes the diffraction allows another alternative reconstruction procedure of a diffraction pattern.

On the basis of integral representation developed was the efficient algorithm for diffraction phenomena modeling, and computer simulation was performed using the properties of mutual compensation of cells joined inside. Also, it was mentioned that the new representation allows considering the size of elementary cells in computer modeling. Application of the new procedure to practical diffraction-related phenomena, like plane wave diffraction on a wire-frame elliptical aperture, improves the processing efficiency without creating any associated artifacts on the field pattern.

\section{References}

1. M. Born, E. Wolf, Principles of Optics, 6-th ed. Pergamon Press, Oxford, (1991).

2. A. Sommerfeld. Mathemathishe theorie der diffraction. // Math. Annalen, 47, pp. 317-374 (1896)

3. Khizhnyak A.I., Anokhov S.P., Lymarenko R.A., Soskin M.S., Vasnetsov M.V. The structure of edge-dislocation wave originated in plane-wave diffraction by a half-plane. // J. Opt. Soc. Am. A. 17(12), pp. 2199-2207 (2000).

4. Anokhov S.P., Khizhnyak A.I., Lymarenko R.A. Diffraction of optical beam by a half-plane. // Sem. Phys. Quant. Elect. \& Opt. 3(1), pp. 94-101 (2000).

5. J.D. Jackson, Classical electrodynamics, John Wiley \& sons, Inc., New York - London (1962).

6. Rubinowicz A. Simple derivation of the Miyamoto-Wolf formula for the vector potential associated with a solution of the Helmholtz equation. // J. Opt. Soc. Am. 52, pp. 717-718 (1962).

7. Ruslan A. Lymarenko, Marat S. Soskin, Anatoliy I. Khizhnyak, Sergey P. Anokhov. The properties and space evolution of hidden singularities in apertured electromagnetic field. // Proc. SPIE, 4403, pp.145-152 (2000).

8. S.Anokhov, R.Lymarenko, A.Khizhnyak. Diffraction an arbitrary tilted plane wave diffraction on a slit // Ukr. Phys. J. 46, №1, pp. 62-64 (2001).

9. Marchand E. W. and Wolf E. Consistent formulation of Kirchhoff's diffraction theory. // J. Opt. Soc. Am. 56, pp. 1712-1722 (1966).

10. R. Vaganov, B. Katzelenbaum. Diffraction theory, M. Nauka (1982). 